\title{
Perceptual chunking and its effect on memory in speech processing: ERP and behavioral evidence
}

\author{
Annie C. Gilbert ${ }^{1,2}{ }^{*+}$, Victor J. Boucher ${ }^{1}$ and Boutheina Jemel ${ }^{2,3}$ \\ 1 'Laboratoire de Sciences Phonétiques, Département de Linguistique et de Traduction, Université de Montréal, Montréal, OC, Canada \\ ${ }^{2}$ Laboratoire de Recherche en Neurosciences et Électrophysiologie Cognitive, Hôpital Rivière-des-Prairies, Montréal, OC, Canada \\ ${ }^{3}$ Centre de Recherche Fernand-Seguin, École d'Orthophonie et d'Audiologie, Université de Montréal, Montréal, OC, Canada
}

\section{Edited by:}

Guillaume Thierry, Bangor University, UK

Reviewed by:

Stanka A. Fitneva, Queen's University, Canada

Benjamin Dering, University of Stirling, UK

Matthew Wagers, University of

California at Santa Cruz, USA

\section{${ }^{*}$ Correspondence:}

Annie C. Gilbert, McGill Language and Memory Laboratory, Department of Psychology, McGill University, 1205 Dr. Penfield Avenue, Montreal, OC H3A 1B1, Canada

e-mail: annie.gilbert@umontreal.ca

\section{${ }^{\dagger}$ Present address:}

Annie C. Gilbert, McGill Language and Memory Laboratory, Department of Psychology, McGill University,

Montreal, QC, Canada
We examined how perceptual chunks of varying size in utterances can influence immediate memory of heard items (monosyllabic words). Using behavioral measures and eventrelated potentials (N400) we evaluated the quality of the memory trace for targets taken from perceived temporal groups (TGs) of three and four items. Variations in the amplitude of the $\mathrm{N} 400$ showed a better memory trace for items presented in TGs of three compared to those in groups of four. Analyses of behavioral responses along with P300 components also revealed effects of chunk position in the utterance. This is the first study to measure the online effects of perceptual chunks on the memory trace of spoken items. Taken together, the N400 and P300 responses demonstrate that the perceptual chunking of speech facilitates information buffering and a processing on a chunk-by-chunk basis.

\section{Keywords: chunking, working memory, prosody, ERP, N400, P300}

\section{INTRODUCTION}

The ability to interpret speech sounds inherently requires that rapidly changing sequences be kept in short-term memory (or "working memory," Baddeley, 2010). However, since short-term memory is limited, one must assume that speech processing operates over some chunk of signal that fits this limited store (Kurby and Zacks, 2008; Ezzyat and Davachi, 2011; Farrell, 2012). On this idea, a number of authors argue that there is a basic perceptual or sensori-motor chunking that groups sequential stimuli (see Graybiel, 1998; Gobet et al., 2001; Terrace, 2001). This differs from the conventional notion of chunking suggested by Miller (1956), which involves a conceptual or semantic recoding of information. Miller's chunking stands as a central concept of cognitive psychology. It is essentially defined as a strategy to enhance memory by grouping items in terms of varying semantic attributes, as in the classic example of letter sequences " $I, B, M, F, B, I, C$, $I, A, I, R, S$ " being recalled in terms of the acronyms "I.B.M., F.B.I., C.I.A., I.R.S." Perceptual chunking, on the other hand, is described by Gobet and Terrace as an automatic perceptual process that is domain-general and that creates groups in sequential stimuli. Such grouping is commonly observed in sequence learning tasks. For instance, in learning and producing novel lists of digits or nonsense syllables, temporal groups (TGs) arise spontaneously. These perceptual chunks or groups are generally marked by characteristic delays or a lengthening of inter-response times at the ends of groups (Terrace, 2001). It is known that perceptual chunks do not tend to exceed four items, which conforms to the capacity limits of short-term serial memory (for an extensive discussion of chunk limits in sequence recall, see Cowan, 2000). Moreover, numerous studies have shown that grouping items in three or four benefits sequence memory (Wickelgren, 1964; Broadbent and Broadbent, 1973; Frankish, 1989, 1995; Hitch et al., 1996; Cowan, 2000; Reeves et al., 2000; Terrace, 2001; Mayberry et al., 2002; Chen and Cowan, 2005; Boucher, 2006). What is less recognized, though, is that temporal grouping marked by length changes also operates in speech production and perception. For instance, a number of studies attests to the production of variably termed "accentual" groups in natural speech that do not tend to exceed four syllables on average (e.g., Dauer, 1983; Martin, 1999; Boucher, 2006), while other studies suggest that listeners detect such groups in speech (Nooteboom, 1997; Christophe et al., 2003, 2004; Boucher, 2006; Gilbert et al., 2010; Gilbert, 2012).

Aside from behavioral observations, a recent study by Gilbert et al. (2010), Gilbert (2012) using event-related potentials (ERPs) has provided more direct evidence of the perceptual chunking of heard speech in terms of TGs. In particular, the study showed that a neural component called closure positive shift (CPS) is evoked by lengthening marks of groups -independently of the intonation patterns or the syntactic-semantic content of utterances. In other words, the evoked CPS response revealed that listeners chunk speech in terms of TGs similar to those 
that appear spontaneously in sequence learning across behaviors (Terrace, 2001). As such, the CPS component is characterized by an incremental negativity over midline central sites followed by a rapid positive deflection. This rising negativity across items in a group is suggestive of a buffering of information that ends with a positive shift corresponding to a group-final mark. The CPS has previously been associated with intonation and semantic-syntactic units (cf. Steinhauer etal., 1999; Steinhauer and Friederici, 2001; Kerkhofs et al., 2009; Bögels et al., 2010; Dilley et al., 2010; Itzhak et al., 2010; Brown etal., 2011; Pauker etal., 2011). Though the new evidence of Gilbert clarifies that CPS can specifically reflect a perceptual chunking of speech in TGs, it remains unclear whether such chunks could be linked to processes of working memory. If indeed this is the case, then one might expect that TGs of differing size would influence listeners' immediate memory of speech.

In assessing this hypothesis, we focus in the present report on how perceived groups in utterances variably affect the memory trace of heard items. In our test, we use an adapted Sternberg task where listeners hear an utterance followed by a target word and are asked to indicate, as fast as possible, whether or not the target was part of the utterance.

First, one should keep in mind that the Sternberg task is a memory scanning paradigm. Results on this task generally show that increasing the number of verbal items in a presented set leads to an increase in response times to given targets by about 30 to $40 \mathrm{~ms}$ per item (Sternberg, 1966). This linear relation between the number of items in a set and response times suggests that participants are scanning the entire content of their working memory before responding. In terms of the Sternberg paradigm, such scanning does not imply activation of every stored item but does imply some process of comparison between a target and a memory set. Only when a match is established is there activation or access to an element in the set leading to a response. Furthermore, the ERP literature on memory scanning has generally focused on two ERP components: the P300 and N400 components (see Wolach and Pratt, 2001; Kutas and Federmeier, 2011 for reviews). Whereas the N400 may mostly relate to the activation of items, research has shown that the P300 is associated with stimulus comparison and classification during the scanning process (Kutas et al., 1977; Magliero et al., 1984). Note that, through the years, the P300 has been known to reflect the sum of different components associated to different mechanism rather than a single response (including the P3a, P3b, and Novelty P3, see Polich, 2007 for a review). Here, we refer to the positive response peaking around $300 \mathrm{~ms}$ from the stimulus onset using the generic label "P300" to avoid taking a stand on the specific underlying processes involved, which fall beyond the scope of the present report (Steiner et al., 2013).

A number of studies have reported that the amplitude of P300 evoked by a target decreases as the size of the memory set increases (see, e.g., Marsh, 1975; Gomer et al., 1976; Karrer et al., 1980; Starr and Barrett, 1987; Pratt etal., 1989; Pelosi etal., 1995; Houlihan etal., 1998; Wolach and Pratt, 2001). P300 latencies have also been related to stimulus classification times (Kutas et al., 1977; Duncan-Johnson and Donchin, 1982; McCarthy and Donchin, 1983; Magliero et al., 1984), though there is some disagreement on this point. Several studies show significant increases in P300 latency for a target when sets increase in size (Marsh, 1975; Gomer etal., 1976; Adam and Collins, 1978; Ford et al., 1979, 1982; Pfefferbaum etal., 1980; Kramer et al., 1986; Starr and Barrett, 1987; Pratt et al., 1989) but some show no significant change beyond sets of two or three items (Pfefferbaum et al., 1980; Pelosi et al., 1992, 1995). In the present report, the $\mathrm{P} 300$ component is specifically used to evaluate effects of perceptual chunking on the scanning of working memory.

As for the N400, though this component was first thought to reflect the degree of semantic integration of an item to a preceding sentence (Kutas and Hillyard, 1980), it is now associated with effort relating to the activation (or accessing) of an element in a presented context (for a review, see Kutas and Federmeier, 2011). More specifically, the amplitude of the N400 varies inversely with the degree of pre-activation of an item: the more restricted a context is, the more an item gets preactivated and the smaller the N400 effect (Kutas and Federmeier, 2011; Amoruso etal., 2013; Strauß etal., 2013). This means that the relative amplitude of the $\mathrm{N} 400$ can index the quality of the memory trace of an item. An item with a strong memory trace would be easier to recall and generate a smalleramplitude N400 than an item with a poorer memory trace. Hence, the N400 may serve to evaluate effects of perceptual chunks of varying size on the quality of the memory trace of heard items.

In view of the general nature of chunking and the above evidence, we hypothesize that listeners perceptually chunk utterances in TGs and that this process links to immediate memory of heard speech. To demonstrate this link, we vary the size of TGs from three to four items. Groupings of three and four are known to affect memory differently; with groups of three having optimal benefits for sequence recall (Cowan, 2000; Boucher, 2006; Mathy and Feldman, 2012). In using a Sternberg task to study these effects, we predict that the accuracy of target recognition, response times, and N400 amplitudes will vary with the size of TGs. Furthermore, as we noted earlier, response times in a typical Sternberg task vary with the number of items in a set and item position is generally expected to have little effect. This is generally interpreted as suggesting that subjects are scanning the entire content of their working memory before responding. However, in our task, the presented stimuli are utterances containing TGs. In these contexts, then, items may be perceived in groups so that a scanning may operate by consecutive chunks. In other words, a match between a target and an item of the first chunk in working memory may lead to an earlier activation compared to a target in a following chunk. If this is the case, then response times to targets may vary in terms of whether they occur in the first or second perceptual group in an utterance, and this may be reflected at the level of P300 characteristics. Taken together, a validation of the above hypotheses provides a way of determining how perceptual chunking can influence working memory in speech processing. 


\section{MATERIALS AND METHODS PARTICIPANTS}

The participants were 16 native speakers of French, aged from 19 to 42 years (mean age $=25.6$ years), who presented normal hearing levels following a standard audiometric screening. All were dominant right-handers (Oldfield, 1971), with no history of substance abuse (other than tobacco smoking), and no history of neurological or psychiatric disorder. All showed normal memory performances on the digit-span test of the WAIS (Wechsler, 1997; overall, average normalized score: 10.16, std dev.: 2.4). Written consent was obtained from every participant, and the present research protocol was approved by the ethics committee of the Hôpital Rivière-des-Prairies (Montréal, QC, Canada).

\section{STIMULI}

The experimental stimuli for the present study consisted of 100 pairs of French utterances and target lexemes. The target lexemes were all monosyllabic nouns matched to the utterance context. Only nouns were used as targets to avoid any confounding effect of lexical class on the retrieval processes (Halgren et al., 2002). The utterances, on the other hand, were made up of monosyllabic lexemes and functors in a similar sentence structure. This structure allowed a speaker to produce the contexts with specific prosodic patterns and TGs, as summarized in Figure 1. A pacing technique (described below) served to control our experimental variables. These variables involved changing TG length (groups of three and four syllables, see blue and red lines in Figure 1) and $T G$ position (first and second group in the utterance, see dark and light shades respectively). The target lexemes were distributed in equal numbers across conditions so that 25 targets were placed in each combination of TG length and TG position (giving four different sets of 25 stimuli). As for the potential effects of syntax, the grammatical function of the TGs was maintained across utterances: the first TG was always a subject NP, and the second a complement to the subject NP (the third TG, which was VP, is not

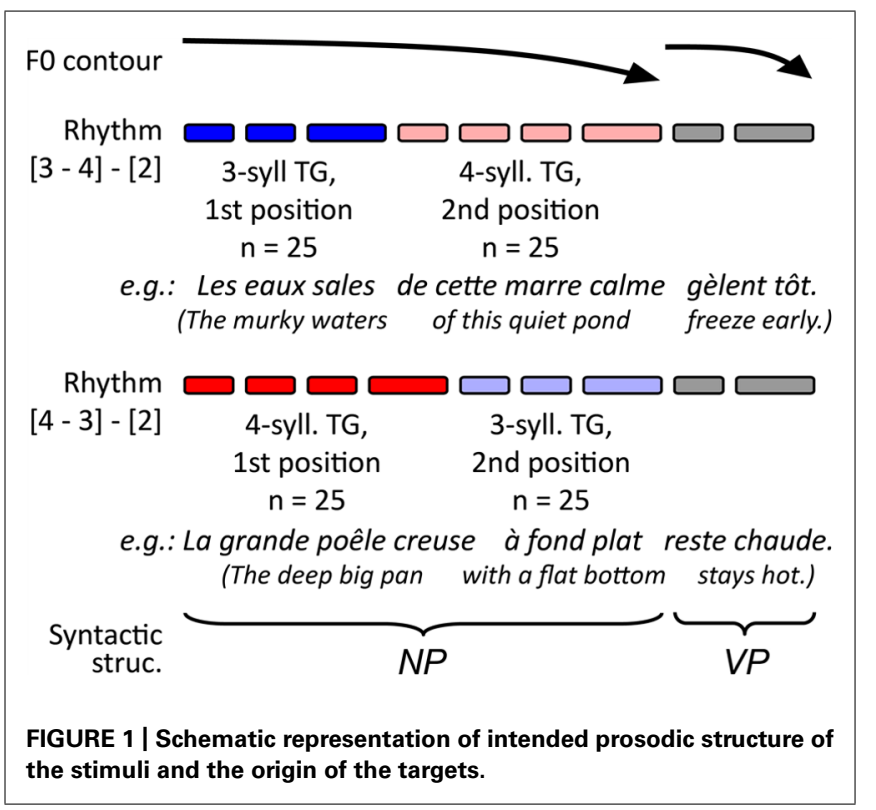

relevant in the present study; the full lists of utterance contexts can be obtained upon request via email to the first author). In all TGs, the target noun was a pre-final element, which served to avoid potential recency effects. Moreover, across conditions, the target nouns used had comparable frequency indices in French $[F(3,94)<0.517 ; \mathrm{MSE}>11,710 ; p>0.672)$, Desrochers, 2006].

In order to create natural sounding contexts, the recording of the target nouns and utterances was done by a male native speaker of French using a pacing technique. By this technique, the speaker follows heard series of rhythmic tones (a metronome-like pacer) in producing utterances with the desired prosody. (For further details of the pacing technique, we refer to Gilbert, 2012). The speaker's productions were recorded using a 16-bit external soundcard (Fast-track Ultra, M-Audio) at a sampling rate of $44.1 \mathrm{kHz}$ and stored in separate wav files. The target lexemes were recorded separately (and not spliced from the original utterance to avoid coarticulation effects) and this provided natural sounding prosody that was appropriate for isolated lexemes in French (Fant et al., 1991). The target lexemes lasted $447.32 \mathrm{~ms}$ on average (minimum $425 \mathrm{~ms}$, maximum $477 \mathrm{~ms}$ ) and bore a neutral tone.

Finally, to tag accurately the onset of target words in the electroencephalography, we relied on the perceptual-center (P-center) of the target lexemes. The P-center constitutes the point in time where a syllable is perceived (Marcus, 1981). Such measures are required to minimize the latency jitter when averaging the ERPs and to allow for precise calculation of intervals between the target and the participant's response. In particular, we made sure that constant intervals were maintained between the P-center of the target lexeme and both the onset and offset of its sound file. In the present case, the $P$-center of the monosyllabic target was always $200 \mathrm{~ms}$ from the beginning and $300 \mathrm{~ms}$ from the end of the file. Audio file editing and amplitude normalization was performed using GoldWave (GoldWave Inc., v5.58).

\section{PROCEDURES}

Figure 2 represents the time-course of a typical trial. All contexts were presented using insert earphones (Eartone 3A, EAR Auditory Systems). The sounds were delivered at a constant intensity, which was calibrated with a sonometer to obtain peak levels of $68 \mathrm{dBA}$ (i.e., a conversational-level sound volume) at the inserts. Participants were instructed to listen to the stimuli while maintaining their gaze at a fixation point on a blank screen. They were also instructed to keep index fingers of both hands on two buttons of a response box. Their task was to indicate, as fast as possible by a key press, whether or not the prompt (the target lexeme) was part of the preceding utterance or not. Half the participants answered affirmatively by pressing the right button with their right hand, half by pressing the left button with their left hand. Sound files were played back via E-prime 1.0 (Psychology Software Tools) in random blocks divided by rest pauses. Each block contained on average 40 stimuli (eight targets and 32 stimuli belonging to different experimental conditions not reported here) delivered in random order with the restriction that no consecutive stimuli presented the same prosodic pattern. The interval between the end of an utterance and the beginning of the audio file of the target lexeme varied from 750 to $1,200 \mathrm{~ms}$ in steps of $50 \mathrm{~ms}$ (11 different intervals). Presentation of successive trials was initiated by 


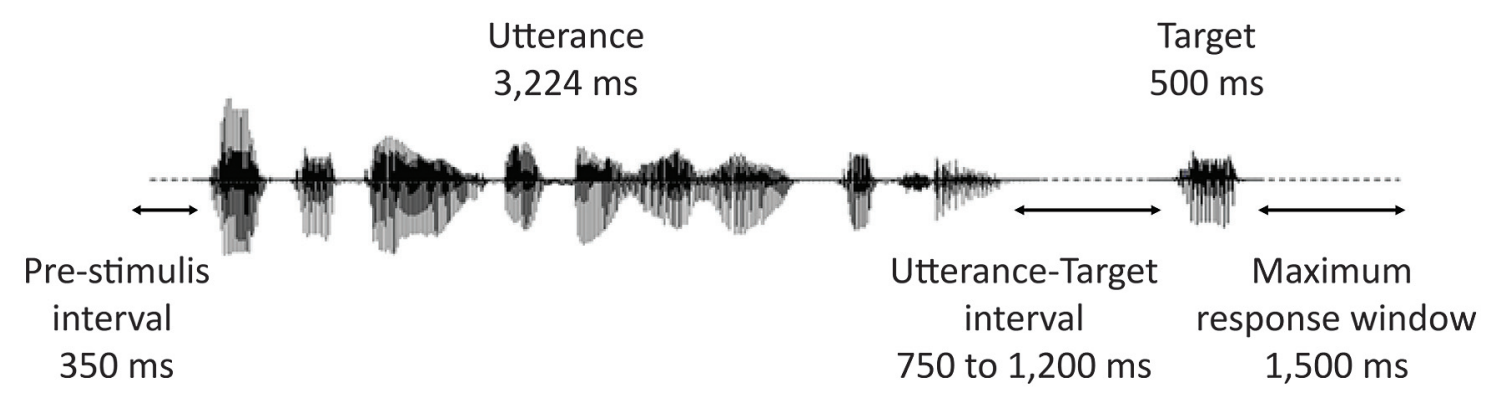

FIGURE 2 | Time-course of a trial.

the participants' response or automatically after $1,500 \mathrm{~ms}$ if no response was given.

\section{EEG RECORDING AND ERP ANALYSIS}

The electroencephalography (EEG) data were recorded through shielded electrodes embedded in an elastic cap (Easy Cap) according to the enhanced 10-20 system (Sharbrough et al., 1991). Two bipolar electrodes placed above and below the dominant eye [vertical electrooculography (EOG)] and at the outer canthus of each eye (horizontal EOG) served to record eye movements and blinks. A left mastoid electrode was used as an online reference for all scalp electrodes, and AFz served as the ground. The EEG including EOG signals were recorded continuously with a bandpass from DC to $100 \mathrm{~Hz}$ at a sampling rate of $512 \mathrm{~Hz}$, and stored with trigger codes. The EEG signal was average-referenced offline and filtered using a $10 \mathrm{~Hz}$ low-pass Butterworth of the sixth order with zero-lag, yielding a $-36 \mathrm{~dB}$ /octave roll-off. Only trials corresponding to correct responses were kept and submitted to the further procedures. EEG segments with eye-blinks and other artifacts were automatically rejected if (i) the standard deviation of the EOG channels within a $200 \mathrm{~ms}$ sliding window exceeds $40 \mu \mathrm{V}$ and if (ii) the standard deviation of any scalp electrode exceeds $20 \mu \mathrm{V}$. Eye blinks were then detected and corrected by subtracting from the EEG the PCA-transformed EOG components for each electrode, weighed according to VEOG propagation factors (computed using linear regression). Segments with other artifacts were rejected.

The obtained artifact-free EEG segments, which were timelocked to the onset of a target sound file, were then averaged for each group length and group position, from $100 \mathrm{~ms}$ before to $700 \mathrm{~ms}$ after the onset of a target sound file. The ERPs were also pooled together according to the TG length irrespective of TG position and vice-versa. This allowed for the calculation of difference waves isolating one factor from the other. Time-sequence topographies of the differential waveforms were used to identify the regions of interest (ROIs). Specifically, we selected the ROIs based upon a visual inspection of the data, taking into account both the individual averages and group average. For the N400 effects, our visual inspection focused on the size of the ERP amplitude difference in the traditional N400 time-window. As for the P300, ROIs were selected based on the sites that showed the largest positive deflections within the P300 time-window. This allowed the selection of the sites showing the most conspicuous N400 and
P300 effects. Each participant's ERP was re-averaged according to the ROIs and these were used to obtain quantitative measures of peak latency and amplitude (maximal and averaged within a time-window). The $\mathrm{N} 400$ was identified as the relative negativity peaking between 300 and 500 ms post-stimulus, (Kutas et al., 1977; Kutas and Federmeier, 2011) over central sites (including FCz, Cz, and $\mathrm{C} 2$ ) whereas the P300 was defined as the positive maximum peaking around $300 \mathrm{~ms}$, immediately before the negative deflexion associated to the N400, over central-parietal sites (including $\mathrm{Cz}$, $\mathrm{C} 1, \mathrm{C} 2, \mathrm{CPz}, \mathrm{CP} 1$, and $\mathrm{CP} 2$ ).

All statistical analyses were computed using $2 \mathrm{X} 2$ analysis of variances (ANOVAS) with TG length (three vs. four) and position in the utterance (first vs. second) as implemented in SPSS (version 17.0).

\section{RESULTS}

\section{BEHAVIORAL DATA}

The recognition task was easy and correct recognition was high, with scores varying from 92 to $96 \%$ correct (see Table 1). A 2X2 ANOVA showed no main effects of TG length or position $\left[F(1,15)<1.561 ; \mathrm{MSE}=0.004 ; p>0.23 ; \eta^{2}<0.095\right]$ and no significant interaction $[F(1,15)=2.529 ; \mathrm{MSE}=0.002 ; p=0.133$; $\left.\eta^{2}=0.144\right]$. As for the reaction times (RTs) for correct responses, a significant main effect was found for the position of the TGs $\left[F(1,15)=9.638 ;\right.$ MSE $\left.=1,149 ; p=0.007 ; \eta^{2}=0.391\right]$, target words pertaining to the first group triggering shorter RT than those pertaining to the second. However, there were no significant

Table 1 | Behavioral results: means and standard deviations of correct recognition scores and $\mathrm{RT}$ as a function of TG length and position.

\section{TG length}

\begin{tabular}{|c|c|c|c|c|}
\hline \multirow[b]{2}{*}{ TG position } & \multicolumn{2}{|c|}{ Three syll. } & \multicolumn{2}{|c|}{ Four syll. } \\
\hline & $\begin{array}{l}\text { Accuracy }(\%) \\
S D\end{array}$ & $\begin{array}{l}\mathrm{RT}(\mathrm{ms}) \\
S D\end{array}$ & $\begin{array}{l}\text { Accuracy }(\%) \\
\text { SD }\end{array}$ & $\begin{array}{l}\mathrm{RT}(\mathrm{ms}) \\
S D\end{array}$ \\
\hline \multirow[t]{2}{*}{ First } & 92.2 & 708.7 & 95.8 & 677.92 \\
\hline & 0.045 & 183.5 & 0.052 & 147.06 \\
\hline \multirow[t]{2}{*}{ Second } & 94.9 & 716.29 & 95.3 & 722.94 \\
\hline & 0.076 & 166.78 & 0.049 & 170.92 \\
\hline
\end{tabular}




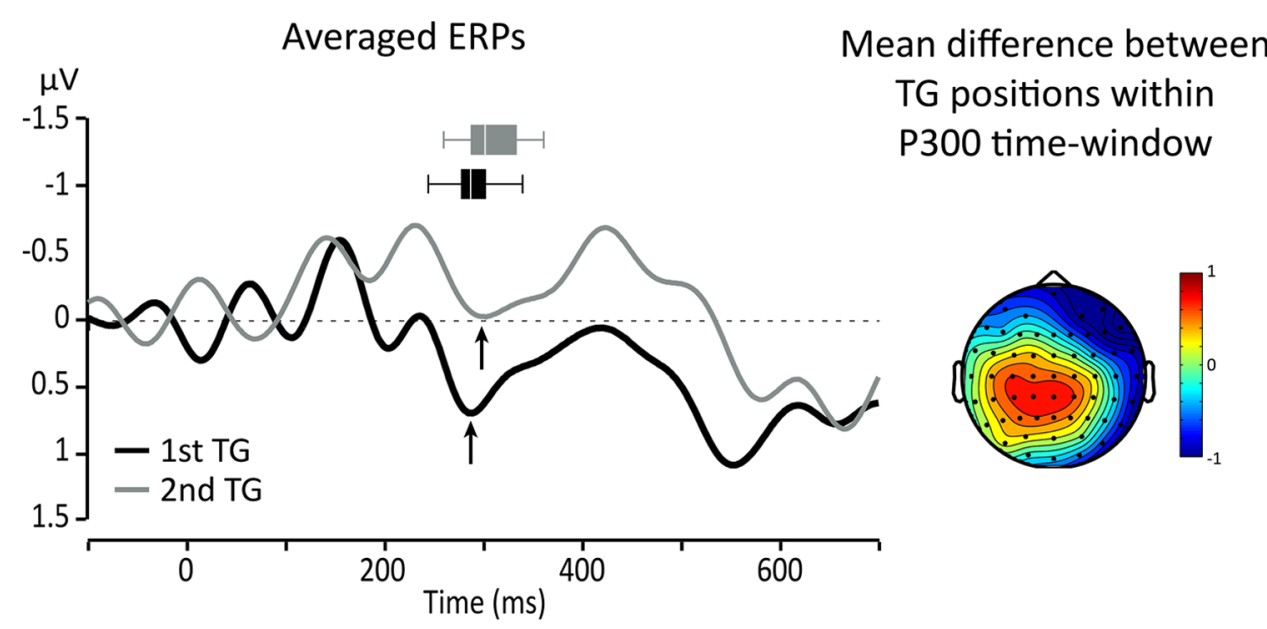

FIGURE 3 | Left: averaged responses for centro-parietal ROI: black line $=$ first TG, gray line $=$ second TG. Arrows indicate the peak of the P300 and horizontal box plots represent peak latency dispersion among participants. Right: topography of the average difference between ERPs for targets from TGs in first and second positions in the utterance. effects of TG length $[F(1,15)=1.047$; MSE $=2,224 p=0.322$; $\left.\eta^{2}=0.065\right]$, and no significant interaction $[F(1,15)=2.142$; $\left.\mathrm{MSE}=2,617 ; p=0.164 ; \eta^{2}=0.125\right]$. Overall, the analyses showed no marked influence of the size of chunks on subjects' behavioral responses, only an effect of TG position in the utterance.

\section{ERP DATA}

The analyses of the continuous EEG focused on two different time-windows previously associated with the P300 and N400 ERPs (described in section 2.4 EEG recording and ERP analysis). Here we present the results obtained for both components separately.

\section{P300}

Since we expected that the P300 would be affected by the position of TGs, topographical maps of the differences between targets from the two TG positions (first and second in the utterance) were used to identify ROI and relevant time-windows. A visual inspection showed that the largest difference was in a central-parietal region which includes $\mathrm{Cz}, \mathrm{C} 1, \mathrm{C} 2, \mathrm{CPz}, \mathrm{CP} 1$, and $\mathrm{CP} 2$. Figure 3 presents topographical ERP difference for TG positions in the time-window of the P300 and the ERP waveforms re-averaged according to the identified ROI (black line $=$ TG in first position, gray line $=$ TG in second position). One can see that the difference between the conditions extends outside the range of the P300. In fact, TGs in second position present, overall, a smaller positivity between 200 and $600 \mathrm{~ms}$ than TGs in the first position (black line).

To evaluate the effect of the experimental conditions on these differences, we focused on the P300 component and measured the average amplitude (per participant) over five consecutive $25 \mathrm{~ms}$ time-windows starting at $200 \mathrm{~ms}$ post-onset. For all of these measures, a 2X2 ANOVA comparing TG length (three- vs. four-syll.) and position (first vs. second) showed no significant main effects $\left[F(1,15)<2.879 ;\right.$ MSE $>1.122 ; p>0.1$; $\left.\eta^{2}<0.161\right]$ or interactions $\left[F(1,15)<3.989 ;\right.$ MSE $\left.>1.064 ; p>0.06 ; \eta^{2}<0.21\right]$. Peak amplitude and latency measures were also taken on the P300 for each participant. The $\mathrm{P} 300$ was identified on the average waveform as the positive peak immediately preceding the negative deflection of the N400 as indicated by the arrows in Figure 3. The results of these latter measures are displayed in Figure 4.

One can see in the left panel of this figure that targets evoke greater P300 amplitudes when they are heard in the first position compared to when they are heard in the second position of the utterance. However, a $2 \mathrm{X} 2$ ANOVA showed that main effects and interactions were not significant $[F(1,15)<3.374$; MSE $>0.907$; $\left.p>0.08 ; \eta^{2}<0.206\right]$. Moreover, what appears as an effect of TG length in the first position was found to be non-significant in terms of a repeated measure $t$-test $[T(1,15)=1.249 ; p=0.231$; $\left.\eta^{2}=0.094\right]$. This likely owes to the small changes in "set sizes" of TGs and the simplicity of the task (see Discussion and Conclusion for an explanation of the seeming discrepancy between the changing neural responses in Figures $\mathbf{3}$ and $\mathbf{4}$ and the statistical results).

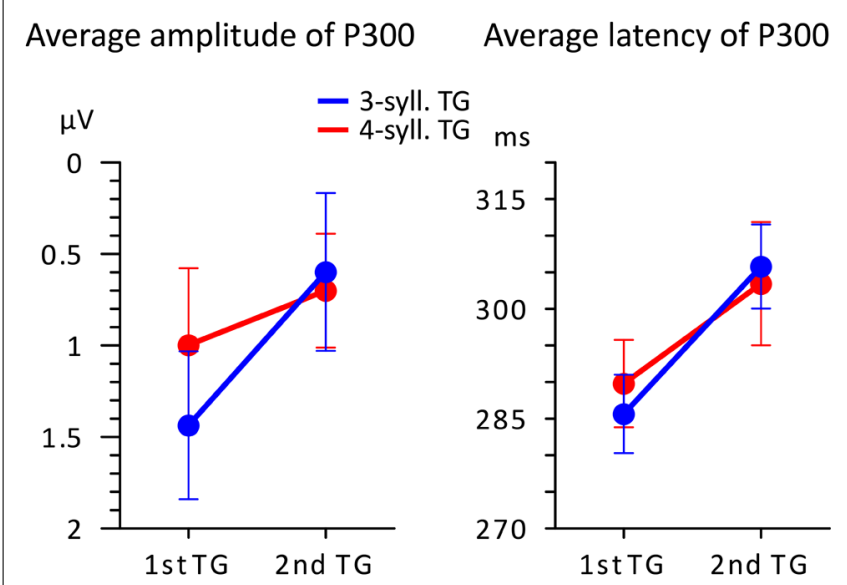

FIGURE 4 | Average amplitude and latency $(n=16)$ of the P300 peak plotted according to TG position (first vs. second) and TG length (blue $=$ 3-syll. vs. red $=$ 4-syll.). Vertical bars represent standard errors. 


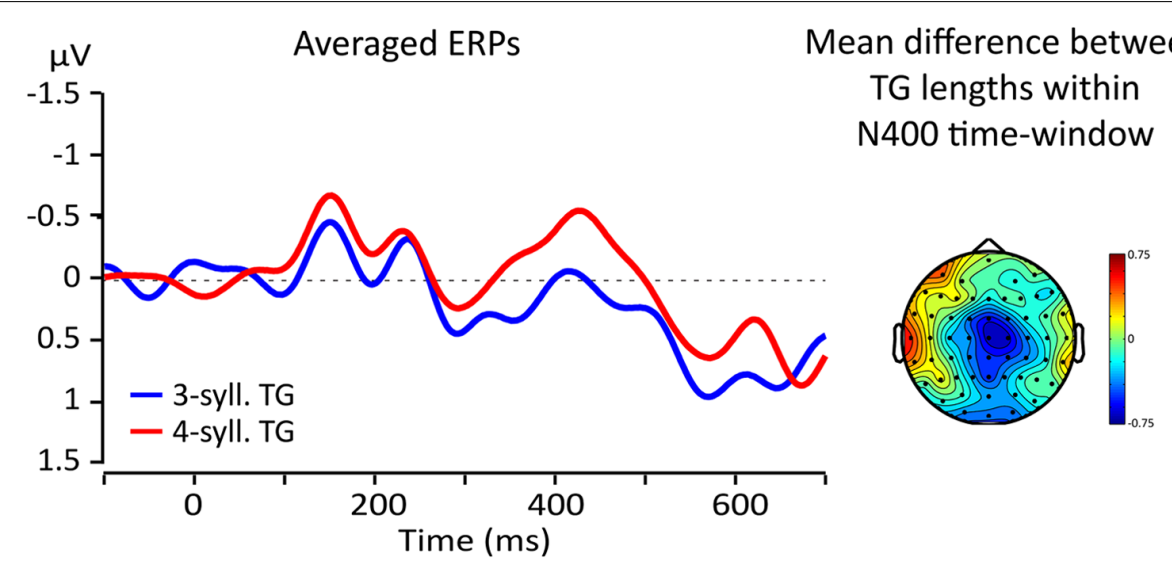

FIGURE 5 | Left: averaged responses for the fronto-central ROI; blue lines = 3-syll TG, red lines = 4-syll TG. Right: topography of the average differences between ERPs for targets from TGs of different lengths (four- minus three-syll.) in the 350-450 ms time-window.

On the other hand, P300 peak latencies (seen in the right panel of Figure 4) present marked differences with respect to position. A 2X2 ANOVA confirmed that only TG position had a significant impact on P300 latency $[F(1,15)=10.865$; $\mathrm{MSE}=0.000$; $\left.p=0.006 ; \eta^{2}=0.455\right]$ with targets from first TGs triggering earlier P300s than targets from the second TGs. The statistical analyses yielded no main effect of TG length and no interactions $\left[F(1,15)<0.098 ;\right.$ MSE $\left.=0.001 ; p>0.759 ; \eta^{2}<0.008\right]$.

\section{N400}

Visual inspections of the topographical difference between TGs of three and four syllables revealed that the greatest difference was found in a region comprising electrodes $\mathrm{FCz}, \mathrm{Cz}$, and C2 (ROI) and at latencies within the usual time-window of the N400 effect (from 300 to 500 ms, Kutas et al., 1977; Kutas and Federmeier, 2011). Figure 5 shows the topographical distribution of the mean amplitude difference between TG lengths (three vs. four) within a $100 \mathrm{~ms}$ time-window from 350 to $450 \mathrm{~ms}$ and the ERPs re-averaged according to the ROI. Line color represents TG length (three-syll. in blue, four-syll. in red). Note that the four-syllable TGs elicit a larger N400 than the three-syllable TGs.

This N400 amplitude difference is further illustrated in Figure 6 representing the mean amplitudes in the $100 \mathrm{~ms}$ time-window according to both TG length and position. This figure illustrates two main findings. First, TGs of three syllables display a smaller negativity than TGs of four syllables when controlling for position. Second, the N400 mean amplitude is greater overall for TGs in the second position than that from first position. Therefore, it seems that two factors influence the amplitude of the N400. However, a 2X2 ANOVA comparing TG length (three- vs. four-syll) and position (first vs. second in utterance) showed a significant effect only for TG length $[F(1,15)=8.506$; $\mathrm{MSE}=0.666 ; p=0.011$; $\left.\eta^{2}=0.362\right]$. Main effects of TG position and interactions were not significant: $\left[F(1,15)<1.152 ; \operatorname{MSE}>0.587 ; p>0.3 ; \eta^{2}<0.071\right]$.

\section{DISCUSSION AND CONCLUSION}

In the above task, listeners heard utterances followed by a word item and were asked to rapidly indicate whether or not the target

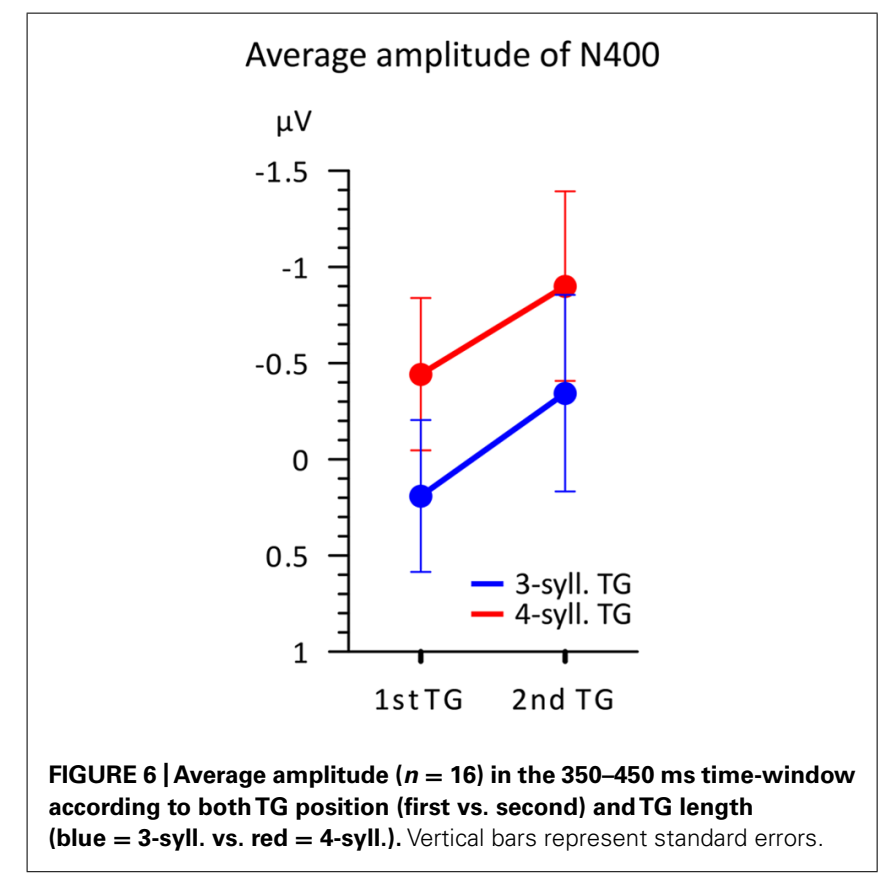

word was part of the heard utterances. Targets within the contexts were placed in perceptual chunks of different size (three or four items) and these chunks appeared in different positions (first or second) in an utterance. The results show that TG size had a significant impact on the quality of the memory trace as reflected in the amplitude of the N400. This effect was not reflected in behavioral responses of recognition accuracy, largely because the task of recalling items in heard utterances can be quite easy, even more so when items are in groups that match the span of working memory (Cowan, 2000). Hence, the task was hardly demanding and this led to high recognition scores with only slight fluctuations across conditions. Nonetheless, the size the TGs had, as predicted, a significant effect on the amplitude of the $\mathrm{N} 400$ component validating the view that perceptual chunks in utterances link to immediate 
memory of speech. Additional effects were studied in terms of the memory scanning task that confirmed that speech in working memory is processed on a chunk-by-chunk basis.

To clarify this last point, it should be recalled that the Sternberg task provides a means of assessing how items are processed in short-term working memory. The above results show significant effects of the position of TGs on RTs. Thus, even though the utterances were of the same length (nine monosyllabic words), the recognition times were shorter when target items were heard in the first group than when they were heard in the second group. To account for such differences, one has to assume that the content of working memory was scanned in terms of ordered chunks and stopped at a point when there was a match between the target and an item in a chunk. Hence, it appears that utterance-related information is stored in serially-ordered chunks and that the scanning of this information in working memory proceeds on a chunk-bychunk basis. Observations of the P300 also appear to support this interpretation, though this component could also be influenced by the location of subject forms in the presented contexts.

The P300 is generally associated with memory scanning processes, and its latency is believed by some to be linked to scanning durations (e.g., Pelosi et al., 1995). In the above observations, P300 latencies show effects of TG position that conform to duration differences in response times: essentially, TGs heard first in the utterances led to shorter response times and earlier P300s compared to later-occurring TGs. It may be useful to mention that these results agree with reports of a relationship between P300 latencies and "set size," where sets in the present experiment are given by TGs (for a review, see Pelosi et al., 1995). That is, the difference with respect to previous work is that the Sternberg task in the present study includes grouped stimuli. As for P300 amplitude, studies have reported decreases in P300 amplitudes for increases in set sizes (for a review, see Wolach and Pratt, 2001). We also observed such patterns of decreasing P300 amplitude, as seen in Figures 3 and 4, though the differences did not reach statistical significance. Yet the results conform to general patterns found across studies: targets heard in TGs that are presented early in a context tend to be associated with larger-amplitude P300s compared to targets heard in later TGs. This seems to be even more marked for targets from short TGs compared to long TGs. The non-significance of the differences likely owes to the relative simplicity of the task or the small difference between our "set sizes" of three and four syllables. Nonetheless, the P300 responses strongly suggest a scanning of working memory on the basis of ordered groups or chunks. It may be noted that this interpretation stands in contrast to a number of behavioral studies involving the recall of visually presented letters or digits, which suggest that position effects on RTs may not reflect a mechanism of serial scanning (e.g., Townsend and Ashby, 1983; McElree and Dosher, 1989). On the other hand, recall of unstructured lists of letters or digits may not extrapolate to the processing of prosodically structured speech where the perception of chunks and memory of serial order is essential to the interpretation of utterances.

Overall, the above results, combined with the earlier findings of Gilbert et al. (2010), Gilbert (2012) offer a perspective on how heard speech is chunked and stored online. Basically, the findings suggest that listeners perceive speech in TGs, and these perceptual chunks are stored in working memory in a serial order. That this perceptual chunking of speech in TGs inherently links to memory processes is demonstrated by the effects of group size on the N400 amplitude. In weighing these findings, it is useful to remark that, despite the large body of research on chunking (for a review, see Cowan, 2000), there is a paucity of work on the role of perceptual chunking in speech processing. This can be explained by the fact that research on chunking is largely based on experiments involving recall whereas perceptual chunking requires a method that captures online responses to incoming stimuli (Terrace, 2001). In this context, the above research using ERPs provides a novel demonstration of how perceptual chunking influences immediate memory of heard speech. It should also be emphasized that the evidence indicating that listeners perceive speech in groups should not be equated with the idea that listeners are detecting prosodic phrases that map syntactic forms (e.g., Frazier et al., 2006). In fact, groups created by delays or a lengthening of elements generally emerge in learning and producing sequences in numerous behaviors both verbal and non-verbal (for examples, see Graybiel, 1998; Terrace, 2001). Hence, these TGs or chunks that arise spontaneously do not "map" syntactic-semantic structure. Instead, they reflect a domain-general sensori-motor chunking that links to working memory (Graybiel, 1998). This presents another perspective on the role of perceptual chunking in language processing that could extend to language learning. In this area, Beckner and Bybee (2009) have expressed the need to explain the emergence of multi-word chunks in language learning by some prelinguistic, domain-general process. The above findings may indicate that perceptual chunking can constitute one of those domain-general processes.

\section{ACKNOWLEDGMENTS}

This research was partly funded by SSHRC grant number 4102008-1732 awarded to Victor J. Boucher and by SSHRC and FQRSC scholarships awarded to Annie C. Gilbert.

\section{REFERENCES}

Adam, N., and Collins, G. I. (1978). Late components of visual evoked potential to search in short term memory. Electroencephalogr. Clin. Neurophysiol. 44, 147-156. doi: 10.1016/0013-4694(78)90261-4

Amoruso, L., Gelormini, C., Aboitiz, F., Alvarez González, M., Manes, F., Cardona, J., et al. (2013). N400 ERPs for actions: building meaning in context. Front. Hum. Neurosci. 7:57. doi: 10.3389/fnhum.2013.00057

Baddeley, A. (2010). Working memory. Curr. Biol. 20, R136-R140.

Beckner, C., and Bybee, J. (2009). A usage-based account of constituency and reanalysis. Lang. Learn. 59, 27-46. doi: 10.1111/j.1467-9922.2009.00534.X

Bögels, S., Schriefers, H., Vonk, W., Chwilla, D. J., and Kerkhofs, R. (2010). The interplay between prosody and syntax in sentence processing: the case of subject- and object-control verbs. J. Cogn. Neurosci. 22, 1036-1053. doi: 10.1162/jocn.2009.21269

Boucher, V. J. (2006). On the function of stress rhythms in speech: evidence of a link with grouping effects on serial memory. Lang. Speech 49, 495-519. doi: $10.1177 / 00238309060490040301$

Broadbent, D. E., and Broadbent, M. H. P. (1973). Grouping strategies in short-term memory for alpha-numeric lists. Bull. Br. Psychol. Soc. 26, 135.

Brown, M., Salverda, A. P., Dilley, L. C., and Tanenhaus, M. K. (2011). Expectations from preceding prosody influence segmentation in online sentence processing. Psychon. Bull. Rev. 18, 1189-1196. doi: 10.3758/s13423-011-0167-9

Chen, Z., and Cowan, N. (2005). Chunk limits and length limits in immediate recall: a reconciliation. J. Exp. Psychol. Learn. Mem. Cogn. 31, 1235-1249. doi: 10.1037/0278-7393.31.6.1235 
Christophe, A., Gout, A., Peperkamp, S., and Morgan, J. (2003). Discovering words in the continuous speech stream: the role of prosody. J. Phon. 31, 585-598. doi: 10.1016/S0095-4470(03)00040-8

Christophe, A., Peperkamp, S., Pallier, C., Block, E., and Mehler, J. (2004). Phonological phrase boundaries constrain lexical access I. Adult data. J. Mem. Lang. 51, 523-547. doi: 10.1016/j.jml.2004.07.001

Cowan, N. (2000). The magical number 4 in short-term memory: a reconsideration of mental storage capacity. Behav. Brain Sci. 24, 87-185. doi: $10.1017 /$ S0140525X01003922

Dauer, R. M. (1983). Stress-timing and syllabe-timing reanalyzed. J. Phon. 11, 51-62.

Desrochers, A. (2006). OMNILEX: une base de données sur le lexique du français contemporain. CLO/OPL 34, 25-34.

Dilley, L. C., Mattys, S. L., and Vinke, L. (2010). Potent prosody: comparing the effects of distal prosody, proximal prosody, and semantic context on word segmentation. J. Mem. Lang. 63, 274-294. doi: 10.1016/j.jml.2010.06.003

Duncan-Johnson, C. C., and Donchin, E. (1982). The P300 component of the eventrelated brain potential as an index of information processing. Biol. Psychol. 14, 1-52. doi: 10.1016/0301-0511(82)90016-3

Ezzyat, Y., and Davachi, L. (2011). What constitutes an episode in episodic memory? Psychol. Sci. 22, 243-252. doi: 10.1177/0956797610393742

Fant, G., Kruckensberg, A., and Nord, L. (1991). Durational correlates of stress in Swedish, French and English. J. Phonet. 19, 351-365.

Farrell, S. (2012). Temporal clustering and sequencing in short-term memory and episodic memory. Psychol. Rev. 119, 223-271. doi: 10.1037/a0027371

Ford, J. M., Pfefferbaum, A., Tinklenberg, J. R., and Kopell, B. S. (1982) Effects of perceptual and cognitive difficulty on P3 and RT in young and old adults. Electroencephalogr. Clin. Neurophysiol. 54, 311-321. doi: 10.1016/00134694(82)90180-8

Ford, J. M., Roth, W. T., Mobs, R. C., Hopkins, W. F., and Kopell, B. S. (1979). Eventrelated potentials recorded from young and old adults during a memory retrieval task. Electroencephalogr. Clin. Neurophysiol. 47, 450-459. doi: 10.1016/00134694(79)90161-5

Frankish, C. (1989). Perceptual organization and precategorical acoustic storage. J. Exp. Psychol. Learn. Mem. Cogn. 15, 469-479. doi: 10.1037/0278-7393.15.3.469

Frankish, C. (1995). Intonation and auditory grouping in immediate serial recall. Appl. Cogn. Psychol. 9, S5-S22. doi: 10.1002/acp.2350090703

Frazier, L., Carlson, K., and Clifton, C. Jr. (2006). Prosodic phrasing is central to language comprehension. Trends Cogn. Sci. 10, 244-249. doi: 10.1016/j.tics.2006.04.002

Gilbert, A. C. (2012). Le chunking perceptif de la parole: Sur la nature du groupement temporel et son effet sur la mémoire immédiate. Montréal: Université de Montréal.

Gilbert, A. C., Boucher, V. J., and Jemel, B. (2010). "Exploring the rhythmic segmentation of heard speech using evoked potentials," Speech Prosody 2010 100334:1-3, Chicago.

Gobet, F., Lane, P. C. R., Croker, S., Cheng, P. C. H., Jones, G., Oliver, I., et al. (2001) Chunking mechanisms in human learning. Trends Cogn. Sci. 5, 236-243. doi 10.1016/S1364-6613(00)01662-4

Gomer, F. E., Spicuzza, R. J., and O’Donnell, R. D. (1976). Evoked potential correlates of visual item recognition during memory scanning tasks. Physiol. Psychol. 4, 61-65. doi: 10.3758/BF03326547

Graybiel, A. M. (1998). The basal ganglia and chunking of action repertoires. Neurobiol. Learn. Mem. 70, 119-136. doi: 10.1006/nlme.1998.3843

Halgren, E., Dhond, R. P., Christensen, N., Van Petten, C., Marinkovic, K., Lewine, J. D., et al. (2002). N400-like magnetoencephalography responses modulated by semantic context, word frequency, and lexical class in sentences. Neuroimage 17 1101-1116. doi: 10.1006/nimg.2002.1268

Hitch, G. J., Burgess, N., Towse, J. N., and Culpin, V. (1996). Temporal grouping effects in immediate recall: a working memory analysis. Q. J. Exp. Psychol. 49, 116-139. doi: 10.1080/713755609

Houlihan, M., Stelmack, R., and Campbell, K. (1998). Intelligence and the effects of perceptual processing demands, task difficulty and processing speed on P300, reaction time and movement time. Intelligence 26, 9-25. doi: 10.1016/S01602896(99)80049-X

Itzhak, I., Pauker, E., Drury, J. E., Baum, S. R., and Steinhauer, K. (2010). Eventrelated potentials show online influence of lexical biases on prosodic processing. Neuroreport 21, 8-13. doi: 10.1097/WNR.0b013e328330251d

Karrer, R., McDonough, B., Warren, C., and Cone, R. (1980). "CNV during memory retrieval by normal and retarded adults," in Motivation, Motor and Sensory
Processes of the Brain: Electrical Potentials, Behavior and Clinical Use, Progress In Brain Research, eds H. H. Kornhuber and L. Deecke (Amsterdam: Elsevier), 668-672.

Kerkhofs, R., Vonk, W., Schriefers, H., and Chwilla, D. J. (2009). Discourse, syntax, and prosody: the brain reveals an immediate interaction. J. Cogn. Neurosci. 19, 1421-1434. doi: 10.1162/jocn.2007.19.9.1421

Kramer, A., Schneider, W., Fisk, A., and Donchin, E. (1986). The effects of practice and task structure on the components of the eventrelated potential. Psychophysiology 23, 33-47. doi: 10.1111/j.1469-8986.1986. tb00590.x

Kurby, C. A., and Zacks, J. M. (2008). Segmentation in the perception and memory of events. Trends Cogn. Sci. 12, 72-79. doi: 10.1016/j.tics.2007.11.004

Kutas, M., and Federmeier, K. D. (2011). Thirty years and counting: finding meaning in the N400 component of the event-related brain potential (ERP). Annu. Rev. Psychol. 62, 621-647. doi: 10.1146/annurev.psych.093008. 131123

Kutas, M., and Hillyard, S. A. (1980). Reading senseless sentences: brain potentials reflect semantic incongruity. Science 207, 203-205. doi: 10.1126/science. 7350657

Kutas, M., McCarthy, G., and Donchin, E. (1977). Augmenting mental chronometry: P300 as a measure of stimulus evaluation time. Science 197, 792-795. doi: $10.1126 /$ science. 887923

Magliero, A., Bashore, T. R., Coles, M. G. H., and Donchin, E. (1984). On the dependence of P300 latency on stimulus evaluation processes. Psychophysiology 21, 171-186. doi: 10.1111/j.1469-8986.1984.tb00201.x

Marcus, S. M. (1981). Acoustic determinants of perceptual center (P-center) location. Percept. Psychophys. 30, 247-256. doi: 10.3758/BF03214280

Marsh, G. R. (1975). Age differences in evoked potentials correlates of a memory scanning process. Exp. Aging Res. 1, 3-16. doi: 10.1080/03610737508257942

Martin, P. (1999). "Intonation of spontaneous speech in French," in Proceedings of the 14th International Conference of Phonetic Sciences. 17-20, San Francisco, CA.

Mathy, F., and Feldman, J. (2012). What's magic about magic numbers? Chunking and data compression in short-term memory. Cognition 122, 346-362. doi: 10.1016/j.cognition.2011.11.003

Mayberry, R. I., Lock, E., and Kazmi, H. (2002). Linguistic ability and early language exposure. Nature 417, 38. doi: 10.1038/417038a

McCarthy, G., and Donchin, E. (1983). "Chronometric analysis of human information processing," in Tutorials in ERP Research: Endogenous Components, eds A. W. K. Gaillard and W. Ritter (Amsterdam: Holland Publications), 251-268.

McElree, B., and Dosher, B. A. (1989). Serial position and set size in short-term memory: the time course of recognition. J. Exp. Psychol. Gen. 118, 346-373. doi: 10.1037/0096-3445.118.4.346

Miller, G. A. (1956). The magical number seven, plus or minus two: some limits on our capacity for processing information. Psychol. Rev. 63, 81-97. doi: $10.1037 / \mathrm{h} 0043158$

Nooteboom, S. G. (1997). “The prosody of speech: melody and rhythm," in Handbook of Phonetic Sciences, eds W. J. Hardcastle and J. Laver (Oxford: Blackwell Publishers), 640-673.

Oldfield, R. C. (1971). The assessment and analysis of handedness: the Edinburgh Inventory. Neuropsychologia 9, 97-113. doi: 10.1016/0028-3932(71)90067-4

Pauker, E., Itzhak, I., Baum, S. R., and Steinhauer, K. (2011). Effects of cooperating and conflicting prosody in spoken english garden path sentences: ERP evidence for the boundary deletion hypothesis. J. Cogn. Neurosci. 23, 2731-2751. doi: 10.1162 /jocn.2011.21610

Pelosi, L., Hayward, M., and Blumhardt, L. D. (1995). Is "memory-scanning" time in the Sternberg paradigm reflected in the latency of event-related potentials? Electroencephalogr. Clin. Neurophysiol. 96, 44-55. doi: 10.1016/0013-4694(94)00163-

Pelosi, L., Holly, M., Slade, T., Hayward, M., Barrett, G., and Blumhardt, L. D. (1992). Wave form variations in auditory event-related potentials evoked by a memory-scanning task and their relationship with tests of intellectual function. Electroencephalogr. Clin. Neurophysiol. 84, 344-352. doi: 10.1016/01685597(92)90087-R

Pfefferbaum, A., Ford, J. M., Roth, W. T., and Kopell, B. S. (1980). Age differences in P3-reaction time associations. Electroencephalogr. Clin. Neurophysiol. 49, 257265. doi: 10.1016/0013-4694(80)90220-5

Polich, J. (2007). Updating P300: an integrative theory of P3a and P3b. Clin. Neurophysiol. 118, 2128-2148. doi: 10.1016/j.clinph.2007.04.019 
Pratt, H., Michalewski, H. J., Barrett, G., and Starr, A. (1989). Brain potentials in a memory-scanning task. I. Modality and task effects on potentials to the probes. Electroencephalogr. Clin. Neurophysiol. 72, 407-421. doi: 10.1016/00134694(89)90046-1

Reeves, C., Schauder, A. R., and Morris, R. K. (2000). Stress grouping improves performance on an immediate serial list recall task. J. Exp. Psychol. Learn. Mem. Cogn. 26, 1638-1654. doi: 10.1037/0278-7393.26. 6.1638

Sharbrough, F., Chatrian, G.-E., Lesser, R. P., Lüders, H., Nuwer, M., and Picton, T. W. (1991). American electroencephalographic society guidelines for standard electrode position nomenclature. J Clin. Neurophysiol. 8, 200-202. doi: 10.1097/00004691-19910400000007

Starr, A., and Barrett, G. (1987). Disordered auditory short term memory in man and event-related potentials. Brain 110, 935-959.

Steiner, G., Barry, R., and Gonsalvez, C. (2013). Can working memory predict target-to-target interval effects in the P300? Int. J. Psychophysiol. 89, 399-408. doi: 10.1016/j.ijpsycho.2013.07.011

Steinhauer, K., Alter, K., and Friederici, A. D. (1999). Brain potentials indicate immediate use of prosodic cues in natural speech processing. Nat. Neurosci. 2, 191-196. doi: 10.1038/5757

Steinhauer, K., and Friederici, A. D. (2001). Prosodic boundaries, comma rules, and brain responses: the closure positive shift in ERP's as a universal marker for prosodic phrasing in listeners and readers. J. Psycholinguist. Res. 30, 267-295. doi: 10.1023/A:1010443001646

Sternberg, S. (1966). High-speed scanning in human memory. Science 153, 652-654. doi: $10.1126 /$ science.153.3736.652

Strauß, A., Kotz, S., and Obleser, J. (2013). Narrowed expectancies under degraded speech: revisiting the N400. J. Cogn. Neurosci. 25, 1383-1395. doi: 10.1162/jocn_a_00389
Terrace, H. S. (2001). "Chunking and serially organized behavior in pigeons, monkeys and humans," in Avian Visual Cognition, ed. R. G. Cook (Medford, MA: Comparative Cognition Press).

Townsend, J. T., and Ashby, G. F. (1983). The Stochastic Modeling of Elementary Psychological Processes. Cambridge: Cambridge University Press.

Wechsler, D. (1997). WAIS-III Administration and Scoring Manual. San Antonio, TX: Psychological Corporation.

Wickelgren, W. A. (1964). Size of rehearsal group and short-term memory. J. Exp. Psychol. 68, 413-419. doi: 10.1037/h0043584

Wolach, I., and Pratt, H. (2001). The mode of short-term memory encoding as indicated by event-related potentials in a memory scanning task with distractions. Clin. Neurophysiol. 112, 186-197. doi: 10.1016/\$1388-2457(00)00501-0

Conflict of Interest Statement: The authors declare that the research was conducted in the absence of any commercial or financial relationships that could be construed as a potential conflict of interest.

Received: 24 September 2013; accepted: 25 February 2014; published online: 19 March 2014.

Citation: Gilbert AC, Boucher VJ and Jemel B (2014) Perceptual chunking and its effect on memory in speech processing: ERP and behavioral evidence. Front. Psychol. 5:220. doi: $10.3389 /$ fpsyg.2014.00220

This article was submitted to Language Sciences, a section of the journal Frontiers in Psychology.

Copyright ( $\odot 2014$ Gilbert, Boucher and Jemel. This is an open-access article distributed under the terms of the Creative Commons Attribution License (CC BY). The use, distribution or reproduction in other forums is permitted, provided the original author(s) or licensor are credited and that the original publication in this journal is cited, in accordance with accepted academic practice. No use, distribution or reproduction is permitted which does not comply with these terms. 